OPEN ACCESS

Edited by:

Mikhail Lebedev,

Duke University, United States

Reviewed by:

Joon-Ho Shin

National Rehabilitation Center,

South Korea

Wei Peng Teo,

Deakin University, Australia

Toshiki Tazoe,

University of Miami, United States

*Correspondence:

Deborah J. Serrien

deborah.serrien@nottingham.ac.uk

Received: 06 April 2017 Accepted: 22 May 2017

Published: 07 June 2017

Citation:

Serrien DJ (2017) Commentary:

Skilled Bimanual Training Drives Motor

Cortex Plasticity in Children With

Unilateral Cerebral Palsy.

Front. Hum. Neurosci. 11:297.

doi: 10.3389/fnhum.2017.00297

\section{Commentary: Skilled Bimanual Training Drives Motor Cortex Plasticity in Children With Unilateral Cerebral Palsy}

\author{
Deborah J. Serrien * \\ School of Psychology, University of Nottingham, Nottingham, United Kingdom
}

Keywords: rehabilitation, pediatric, transcranial magnetic stimulation, neuroplasticity, hemiplegia

\section{A commentary on}

\section{Skilled Bimanual Training Drives Motor Cortex Plasticity in Children With Unilateral Cerebral} Palsy

by Friel, K. M., Kuo, H. C., Fuller, J., Ferre, C. L., Brandão, M., Carmel, J. B., et al. (2016). Neurorehabil. Neural Repair 30, 834-844. doi: 10.1177/1545968315625838

Unilateral spastic cerebral palsy (USCP) is a childhood-onset neurological disorder that is characterized by motor dysfunction to primarily one side of the body, reducing hand function of the affected hand, and bimanual coordination patterns (Staudt, 2010). To improve manual performance, effective motor rehabilitation techniques are crucial. However, optimization of the treatment methods is challenged by the heterogeneity of the clinical symptoms of children with USCP (Reid et al., 2015). Two main approaches currently exist: (1) constraint-induced movement therapy that involves physical restraint of the less affected upper extremity alongside practice of the more affected upper extremity, and (2) bimanual training therapy that consists of intensive practice of functional activities with both upper extremities. Therefore, the focus of these methods is distinct, although both use training intensity and progression of skill complexity as core characteristics of their programme. This rationale is based on the premise that practice with increased task difficulty drives plasticity of the motor system (Adkins et al., 2006).

In their study, Friel et al. (2016) examine the impact of bimanual training therapy upon manual performance and motor cortex plasticity in children with USCP. To this end, the authors compared structured skill training vs. unstructured hand use with each group of children having $90 \mathrm{~h}$ of bimanual therapy that were spread over 3 weeks of practice. The structured practice group received the Hand-Arm Bimanual Intensive Therapy (Charles and Gordon, 2006), which included the following key components: increase of task difficulty, practice of component movements that are part of a more complex task, and training of functional goals. In contrast, the unstructured practice group engaged in bimanual activities but did not train skillful movements or functional goals. Both groups were evaluated by outcome measures that quantified unimanual dexterity (Jebsen et al., 1969), bimanual performance (Krumlinde-Sundholm et al., 2007), and functional goal performance (Law et al., 1990). The authors further used single-pulse transcranial magnetic stimulation (TMS) to map the representation and excitability of the digit and wrist muscles of the affected hand at three time points: before, immediately after and 6 months after training. Combined, these measurements that assess cortical changes alongside motor skill improvements represent a powerful means to study how the brain responds to training. 
The data showed that structured and unstructured training enhanced bimanual performance as well as hand dexterity of the affected hand; an improvement that was preserved at 6 month post-practice. Conversely, only the group that received structured training showed a significant increase in the size of the motor map of the affected hand and amplitude of the motor responses to TMS. This underlines the importance of skilled practice such as intricate bimanual activities as a critical factor for triggering cortical plasticity. However, despite improved hand function as a result of the intervention, little is known about the strengthening of the bimanual coordination pattern per se. In particular, although bimanual training therapy is aimed at augmenting the coordination of both upper limbs, the outcome measures focus on the functional improvement of the affected hand for tasks that require both hands rather than on measuring the process that characterizes the bimanual demands. In this respect, the coordinated use of both hands typically require an accurate spatio-temporal integration of each hand; an ability that develops with age (Fagard et al., 2001; Serrien et al., 2014; Babik and Michel, 2016) and that especially becomes apparent when performing daily-life activities during which the action of one hand is supported by a cooperative action from the other hand. Therefore, it is argued that the investigation of the bimanual synergy in relation to the individual task components could ascertain additional measurement outcomes, identify distinct time scales of motor learning (Serrien, 2008) and clarify interactions between levels of motor organization that occur as a result of rehabilitation.

The findings further revealed that the changes in cortical plasticity for the structured practice group were independent of the hemisphere of control, i.e., contralateral or ipsilateral to the affected hand. This suggests that both hemispheres responded to the bimanual training; an observation that contrasts with data from the constraint-induced movement therapy that have shown that functional gains are less pronounced in children with ipsilateral than contralateral control of the affected hand (Kuhnke et al., 2008). Taking into account that hand function in cerebral palsy is influenced by the nature of the corticospinal projections

\section{REFERENCES}

Adkins, D. L., Boychuk, J., Remple, M. S., and Kleim, J. A. (2006). Motor training induces experience-specific patterns of plasticity across motor cortex and spinal cord. J. Appl. Physiol. 101, 1776-1782. doi: 10.1152/japplphysiol.0051 5.2006

Babik, I., and Michel, G. F. (2016). Development of role-differentiated bimanual manipulation in infancy: part 1. The emergence of the skill. Dev. Psychobiol. 58, 243-256. doi: 10.1002/dev.21382

Charles, J., and Gordon, A. M. (2006). Development of hand-arm bimanual intensive training (HABIT) for improving bimanual coordination in children with hemiplegic cerebral palsy. Dev. Med. Child Neurol. 48, 931-936. doi: $10.1017 /$ S0012162206002039

Fagard, J., Hardy-Léger, I., Kervella, C., and Marks, A. (2001). Changes in interhemispheric transfer rate and the development of bimanual coordination during childhood. J. Exp. Child Psychol. 80, 1-22. doi: 10.1006/jecp.2000. 2623

Friel, K. M., Kuo, H. C., Fuller, J., Ferre, C. L., Brandão, M., Carmel, J. B., et al. (2016). Skilled bimanual training drives motor cortex plasticity in children
(Holmström et al., 2010), it is essential that training interventions are optimized across the pathology of USCP.

That the children who showed the strongest functional improvement also demonstrated the largest expansion of the cortical motor map points to neuroplasticity as a result of rehabilitation and is in line with the existence of reorganization mechanisms (Mackey et al., 2014). Thus, the present findings highlight the importance of studying brain-behavioral adaptations in children with USCP by using various experimental tools, tests, and time points during training interventions. This argument is especially relevant considering that the children who were part of the unstructured practice group improved their hand function with training but did not show modulations in the size of their cortical motor map within the time frame of investigation. Notwithstanding this clinical significance, corticospinal, and interhemispheric motor systems have not been fully studied in the developing brain. Therefore, detailing the properties of the motor circuits in childhood and adolescence will further enhance the evaluation of rehabilitation interventions for USCP.

Taken together, the study by Friel et al. (2016) provides exciting research that exposes the significance of skilled bimanual training for steering the interplay between neural changes and behavioral improvements in children with USCP. Further work that examines cortical plasticity across different motor systems and time scales alongside the development of measurements that assess coordination outcomes will be important to enhance insights into the neural recalibration that takes place in response to bimanual training. Due to advances in experimental acquisition and analysis techniques, future research will permit to detail how training with the aim to improve hand function in USCP shapes the brain and the mechanisms that support neuroplasticity.

\section{AUTHOR CONTRIBUTIONS}

The author confirms being the sole contributor of this work and approved it for publication.

with unilateral cerebral palsy. Neurorehabil. Neural Repair 30, 834-844. doi: $10.1177 / 1545968315625838$

Holmström, L., Vollmer, B., Tedroff, K., Islam, M., Persson, J. K., Kits, A., et al. (2010). Hand function in relation to brain lesions and corticomotor-projection pattern in children with unilateral cerebral palsy. Dev. Med. Child Neurol. 52, 145-152. doi: 10.1111/j.1469-8749.2009.03496.x

Jebsen, R. H., Taylor, N., Trieschmann, R. B., Trotter, M. J., and Howard, L. A. (1969). An objective and standardized test of hand function. Arch. Phys. Med. Rehabil. 50, 311-319.

Krumlinde-Sundholm, L., Holmefur, M., Kottorp, A., and Eliasson, A. C. (2007). The Assisting Hand Assessment: current evidence of validity, reliability, and responsiveness to change. Dev. Med. Child Neurol. 49, 259-264. doi: 10.1111/j.1469-8749.2007.00259.x

Kuhnke, N., Juenger, H., Walther, M., Berweck, S., Mall, V., and Staudt, M. (2008). Do patients with congenital hemiparesis and ipsilateral corticospinal projections respond differently to constraint-induced movement therapy? Dev. Med. Child Neurol. 50, 898-903. doi: 10.1111/j.1469-8749.2008.03119.x

Law, M., Baptiste, S., McColl, M., Opzoomer, A., Polatajko, H., and Pollock, N. (1990). The Canadian Occupational Performance Measure: an outcome 
measure for occupational therapy. Can. J. Occup. Ther. 57, 82-87. doi: 10.1177/000841749005700207

Mackey, A., Stinear, C., Stott, S., and Byblow, W. D. (2014). Upper limb function and cortical organization in youth with unilateral cerebral palsy. Front. Neurol. 5:117. doi: 10.3389/fneur.2014.00117

Reid, L. B., Rose, S. E., and Boyd, R. N. (2015). Rehabilitation and neuroplasticity in children with unilateral cerebral palsy. Nat. Rev. Neurol. 11, 390-400. doi: 10.1038/nrneurol.2015.97

Serrien, D. J. (2008). Coordination constraints during bimanual versus unimanual performance conditions. Neuropsychologia 46, 419-425. doi: 10.1016/j.neuropsychologia.2007.08.011

Serrien, D. J., Sovijärvi-Spapé, M. M., and Rana, G. (2014). Developmental changes in motor control: insights from bimanual coordination. Dev. Psychol. 50, 316-323. doi: 10.1037/a0032996
Staudt, M. (2010). Brain plasticity following early life brain injury: insights from neuroimaging. Semin. Perinatol. 34, 87-92. doi: 10.1053/j.semperi.2009. 10.009

Conflict of Interest Statement: The author declares that the research was conducted in the absence of any commercial or financial relationships that could be construed as a potential conflict of interest.

Copyright (C) 2017 Serrien. This is an open-access article distributed under the terms of the Creative Commons Attribution License (CC BY). The use, distribution or reproduction in other forums is permitted, provided the original author(s) or licensor are credited and that the original publication in this journal is cited, in accordance with accepted academic practice. No use, distribution or reproduction is permitted which does not comply with these terms. 